\title{
INVESTIGATION OF THE NONLINEAR FLUTTER OF VISCOELASTIC PLATES IN A SUPERSONIC FLOW
}

\author{
Bakhtiyar Khudayarov \\ Tashkent institute of irrigation and melioration. \\ UZBEKISTAN, 700135, Tashkent-135, Chilanzar-16-24-56. \\ E-mail: bakht-flpo@yandex.ru
}

\begin{abstract}
In this paper the flutter of nonlinear viscoelastic plates in a supersonic flow is investigated. The basic direction of work is consisted in taking into account of viscoelastic material's properties at supersonic speeds.Quasisteady aerodynamic panel loadings are determined using piston theory. The vibration equations relatively of deflection are described by Integro-differential equations in partial derivatives. The plate nonlinear partial integro-differential equation is transformed info a set of nonlinear ordinary IDE through a Bubnov-Galerkin's approach. The resulting system of IDE is solved through the Badalov-Eshmatov integration method. Critical speeds for plate flutter are defined.
\end{abstract}

Keywords: viscoelastic, flutter, plate, Integro-differential equation

\section{INTRODUCTION}

At present, there are much literature on the flutters, and the problem sufficiently is solved, though we need enough accordance between the theory and experiments for configuration and Mach number, In the $[1,2]$ work Fung has analyzed the problem of the flutters. The problem is investigated by Dowell and Boss [3].

The stability of plates and shells in supersonic flow was considered in many works [4-6]. The analysis of the motion of structures under deterministic treatment of the problem is given in these works. The mathematical modeling is based on linear representation of elastic and aerodynamic forces. The aerodynamic load is obtained from a quasi-steady first-order aerodynamic piston theory.

In connection with wide introduction of composite materials in the aircraft designs, creation of the calculation methods of separate elements of constructions, made of the materials had great importance [7, 8].

In the given work mathematical models of the problems of the viscous-elastic plates were constructed by taking account of the geometrical and aero dynamical non-linearity, aero dynamical damping, statically pressure dropping on the basis of the Kirchhoff-Love hypothesis.

For the solution to the system was applied the problem was solved by the usual IDE based on flexure polynomial approximation by means of Bubnov-Galerkin's method. The numerical method based on quatrature proposed by F.Badalov and
H.Eshmatov [9]. It was described the algorithm of the numerical solution on the basis of the method.

Critical speeds of the viscous-elastic plate's flutter moved by constant super sound speed were found in all parts of the problem.

\section{MAIN EQUATIONS}

Considered here is the response of three dimensional viscous-elastic plates in high supersonic flow to a disturbance.

Von Karman's large deflection plate equations are used to describe the viscous-elastic behavior of the plate [4]:

$$
\begin{aligned}
& \frac{D}{h}\left(1-R^{*}\right) \nabla^{4} W=L(W, \phi)-\rho \frac{\partial^{2} W}{\partial t^{2}}+\frac{q}{h} \\
& \frac{1}{E} \nabla^{4} \phi=-\frac{1}{2}\left(1-R^{*}\right) L(W, W) .
\end{aligned}
$$

Where $D$ is the plate stiffness obtained from $D=E h^{3} / 12\left(1-\mu^{2}\right)$, with $E$ being the Young modulus, $h$ the plate thickness, and $\mu$ the Poisson ratio. In Eq.(1), $\rho$ is the plate material density. The biharmonic term is defined as

$$
\nabla^{4}=\frac{\partial^{4}}{\partial x^{4}}+2 \frac{\partial^{4}}{\partial x^{2} \partial y^{2}}+\frac{\partial^{4}}{\partial y^{4}} .
$$

In Eq.(1), $R^{*}$ is the relaxation operator obtained from 


$$
R^{*} \psi=\int_{0}^{t} R(t-\tau) \psi(\tau) d \tau
$$

$R(t-\tau)$ the relaxation kernel. The plate deflection is $W$ whereas $\phi$ is the Airy stress function.

In Eq.(1), $q$ is the aerodynamic pressure loading due to the uniform supersonic flow. For high Mach numbers this pressure is given approximately by the usual quasi-steady expression

$$
q=-\mathrm{B} \frac{\partial W}{\partial t}+B V \frac{\partial W}{\partial x}-B_{1} V^{2}\left(\frac{\partial W}{\partial x}\right)^{2} .
$$

Where

$$
\mathrm{B}=æ \frac{p_{\infty}}{V_{\infty}} ; \mathrm{B}_{1}=æ(æ+1) \frac{p_{\infty}}{4 V_{\infty}^{2}} ;
$$

$p_{\infty}$-pressure; $V_{\infty}$ is the speed of sound.

\section{SOLUTION OF THE STRESS FUNCTION}

To solve Eq.(2) for the function $\phi$, the deflection $W(x, y, t)$ may be expressed in terms of the assumed mode expansion

$$
W(x, y, t)=\sum_{n=1}^{\infty} \sum_{n=1}^{\infty} W_{n m}(t) \sin (n \pi x / a) \sin (m \pi y / b),
$$

where $W_{n m}(t)$ are the generalized coordinates.

Solution (4) satisfies the geometric boundary conditions of a simply supported plates at the four sides. Substitution of Eq.(4) into Eq.(2) gives the non-homogeneous partial differential equation

$$
\begin{aligned}
& \nabla^{4} \phi=\frac{E \pi^{4}}{4 a^{2} b^{2}} \sum_{n, i=1 n, r=1}^{\infty} \sum_{n}^{\infty}\left(1-R^{*}\right) W_{n m}(t) W_{i r}(t) n r \times \\
& \times\left\{(i r-n m) \lambda_{1}+(n m+i r) \lambda_{2}+(n m+i r) \lambda_{3}+(i r-n m) \lambda_{4}\right\}
\end{aligned}
$$

where

$$
\begin{aligned}
& \lambda_{1}=\operatorname{Cos} \frac{(n+i) \pi x}{a} \operatorname{Cos} \frac{(m+r) \pi y}{b} ; \\
& \lambda_{2}=\operatorname{Cos} \frac{(n+i) \pi x}{a} \operatorname{Cos} \frac{(m-r) \pi y}{b} ; \\
& \lambda_{3}=\operatorname{Cos} \frac{(n-i) \pi x}{a} \operatorname{Cos} \frac{(m+r) \pi y}{b} ; \\
& \lambda_{4}=\operatorname{Cos} \frac{(n-i) \pi x}{a} \operatorname{Cos} \frac{(m-r) \pi y}{b} .
\end{aligned}
$$

The solution of this equation consists of two parts: the particular integral and the homogeneous solution. The particular integral may be written in the from

$$
\phi(x, y, t)=E \sum_{n, i=m, r=1}^{\infty} \sum^{\infty}\left(1-R^{*}\right) W_{n m}(t) W_{i v}(t)\left[A_{n m i} \lambda_{2} B_{n m i} \lambda_{4}+C_{n m i} \lambda_{1}+D_{n m i} \gamma_{3}\right]
$$

where

$$
\begin{gathered}
A_{n m i r}=\frac{\lambda^{2} n m(n m+i r)}{4\left[(n+i)^{2}+\lambda^{2}(m-r)^{2}\right]^{2}} ; \\
B_{n m i r}=\frac{\lambda^{2} n m(i r-n m)}{4\left[(n-i)^{2}+\lambda^{2}(m-r)^{2}\right]^{2}} ; \\
C_{n m i r}=\frac{\lambda^{2} n m(i r-n m)}{4\left[(n+i)^{2}+\lambda^{2}(m+r)^{2}\right]^{2}} ; \\
D_{n m i r}=\frac{\lambda^{2} n m(n m+i r)}{4\left[(n-i)^{2}+\lambda^{2}(m+r)^{2}\right]^{2}} ; \lambda=\frac{a}{b} .
\end{gathered}
$$

\section{MODAL DYNAMIC EQUATIONS}

Substituting the stress function solution (6) are substituted along with the modal expansion (4) for $W$ into the plates equation of motion (1) and applying Bubnov-Galerkin method gives the ordinary nonlinear IDE :

$$
\begin{aligned}
& \rho \ddot{W}_{k l}+\frac{E h^{2} \pi^{2}}{b^{4}}\left\{\frac{\pi^{2}}{12\left(1-\mu^{2}\right)}\left[\left(\frac{k}{\lambda}\right)^{2}+l^{2}\right]\right\}\left(1-R^{*}\right) W_{k l}= \\
& =-\frac{B}{h} \dot{W}_{k l}-\frac{64}{\pi^{4}} \frac{\rho_{c} V_{\infty}^{2}}{d} \gamma_{k} \gamma_{l} \sum_{n=1}^{\infty} \sum_{m=1}^{\infty} \gamma_{n} \gamma_{m} W_{n m}+\frac{16 \alpha_{k} \alpha_{l} \Delta p}{k l h \pi^{2}}- \\
& -\frac{E \pi^{2}}{b^{4}} \sum_{n, i, j=1}^{\infty} \sum_{m, r, s=1}^{\infty} a_{k l n m i r j} W_{n m}\left(1-R^{*}\right) W_{i r} W_{j s}+ \\
& +\frac{2 B V}{h a} \sum_{n=1}^{\infty} \gamma_{k l} W_{n l}-\frac{B_{1} V^{2}}{h a^{2}} \sum_{n, i=1}^{\infty} \sum_{m, r=1}^{\infty} G_{k l n m i r} W_{n m} W_{i r}
\end{aligned}
$$

where

$$
\begin{aligned}
& \gamma_{k n}=n\left(\gamma_{n+k}-\gamma_{n-k}\right) ; \\
& \gamma_{k}= \begin{cases}\frac{\alpha_{k}}{k}, & \mathrm{k}-\text { odd } \\
0, & \mathrm{k}=0 \text { or } \mathrm{k}-\text { even, }\end{cases}
\end{aligned}
$$

$a_{k l n m i r j s}, G_{k l n m i r}$ - non-dimensional coefficients [10].

Introducing these parameters,

$$
\frac{W}{h} ; \frac{\mathrm{V}_{\infty} t}{a} ; \frac{a}{\mathrm{~V}_{\infty} t} R(t)
$$

and keeping the previous designations, the equations (7) takes the non-dimensional forms.

Equation (7) takes the non-dimensional form 


$$
\begin{aligned}
& \ddot{W}_{k l}+\delta_{1} \sum_{n, i, j=1}^{\infty} \sum_{m, r, s=1}^{\infty} a_{k \mathrm{n} m i r j s} W_{n m}\left(1-R^{*}\right) W_{i r} W_{j s}+ \\
& +\omega_{1 k l}\left(1-R^{*}\right) W_{k l}+M \dot{W}_{k l}-\delta_{2} \sum_{n=1}^{\infty} \gamma_{k l} W_{n l}-\omega_{3 k l}+ \\
& +\delta_{3} \sum_{n, i=1}^{\infty} \sum_{m, r=1}^{\infty} G_{k \mathrm{ln} m i r} W_{n m} W_{i r}+\omega_{2 k l} \sum_{n=1}^{\infty} \sum_{m=1}^{\infty} \gamma_{n} \gamma_{m} W_{n m}=0,
\end{aligned}
$$

where

$$
\begin{gathered}
\Omega^{2}=\frac{\pi^{4}}{12\left(1-\mu^{2}\right)} M_{E}^{2}\left(\frac{h}{a}\right)^{2}, M=æ M_{p}^{2}\left(\frac{a}{h}\right), \\
\mathrm{M}_{1}=æ(æ+1) \frac{M_{p}^{2}}{4} ; \\
M^{*}=\frac{V}{V_{\infty}}-\text { Mach number; } \\
M_{E}=\sqrt{\frac{E}{\rho V_{\infty}^{2}}} ; \quad M_{p}=\sqrt{\frac{P_{\infty}}{\rho V_{\infty}^{2}}} \\
\omega_{1 k l}=\lambda^{4} \Omega^{2}\left[\left(\frac{k}{\lambda}\right)^{2}+l^{2}\right]^{2} ; \\
\delta_{1}=\frac{12 \lambda^{4}\left(1-\mu^{2}\right) \Omega^{2}}{\pi^{2}} ; \delta_{2}=2 M M^{*} ; \\
\delta_{3}=M_{1} M^{*^{2}} .
\end{gathered}
$$

Equations (8) allow conducting qualitative analysis of solving the problems of the flutters of the viscous-elastic plates.

\section{NUMERICAL RESULTS}

Integration of the system (8) of KoltunovRjanitsin kernel $\left(R(t)=A \exp (-\beta t) t^{\alpha-1}, 0<\alpha<1\right)$ was made by numerical method [9]. According to this method the equation (8) twice integrating over $t$ from 0 to $t$. By considering for that purpose $t=t_{i}$, $t_{i}=i h, i=1,2, \ldots$ ( $h=$ const) and replacing integrals by quadrature formulas of trapezes for calculation of $W_{i k l}=W_{k l}\left(t_{i}\right)$ we shall receive recurrent formulas.

On the basis of algorithm, is created of the

\begin{tabular}{|c|c|c|c|c|c|}
\hline A & $\alpha$ & $\beta$ & $\lambda$ & $a / h$ & $V_{c r}$ \\
\hline \begin{tabular}{|c|}
0 \\
0,001 \\
0,04 \\
0,1 \\
\end{tabular} & 0,25 & 0,05 & 3 & 400 & $\begin{array}{c}854,15 \\
852,45 \\
762 \\
753 \\
\end{array}$ \\
\hline 0,05 & $\begin{array}{l}0,1 \\
0,5 \\
0,75\end{array}$ & 0,05 & 3 & 400 & $\begin{array}{l}624 \\
835 \\
863\end{array}$ \\
\hline 0,1 & 0,25 & \begin{tabular}{|c|}
0,08 \\
0,1 \\
\end{tabular} & 3 & 400 & $\begin{array}{l}762 \\
746 \\
\end{array}$ \\
\hline 0,1 & 0,25 & 0,05 & $\begin{array}{c}1 \\
1,5 \\
2\end{array}$ & 400 & \begin{tabular}{|c|}
488 \\
839 \\
1363 \\
\end{tabular} \\
\hline 0,1 & 0,25 & 0,05 & 3 & $\begin{array}{l}350 \\
450 \\
500\end{array}$ & $\begin{array}{c}1130 \\
525 \\
435\end{array}$ \\
\hline
\end{tabular}
programs in algorithmic language "Turbo-Pascal".

The results of calculations are given in Table.

The results of calculations, conducted by the formulae (8) for plates, flowed by supersonic gas with parameters $p_{\infty}=1,014 \mathrm{~kg} / \mathrm{sm}^{2}, \varkappa^{2}=1,4$ and $V_{\infty}=340 \mathrm{~m} / \mathrm{s}$ are given in Table.
Table. Dependences of critical speeds of visco-elastic plate from physico- mechanical and geometrical parameters

As a criterion, determining the critical speed $V_{c r}$ we take condition that at the speeds amplitude of oscillations is changed by harmonica law. At more supercritical speeds oscillatory motions take place in intensive amplitude, which can lead to damage of the construction. At $V<V_{c r}$ the oscillatory amplitude is attenuated.

The analysis of the results given in the table shows that the coefficient $V_{c r}$ is elastic $(A=0)$ and viscous-elastic $(A=0,1)$ according to $854,15 \mathrm{~m} / \mathrm{s}$ and $753 \mathrm{~m} / \mathrm{s}$. Viscous-elastic properties of the material lead to reduction of the flutter speed.

Increasing the $\alpha$ parameter leads to changing $V_{c r}$ . The research was conducted at $\alpha=0,1 ; 0,5 ; 0,75$. It's clear that increasing the parameter $\alpha$ from 0,1 to 0,75 is accompanied by increasing the critical number $V_{c r}$ of the flutter from $V_{c r}=624 \mathrm{~m} / \mathrm{s}$ to $V_{c r}=863 \mathrm{~m} / \mathrm{s}$.

It was observed the influence of the parameter $\lambda$ to plates. Increasing the parameter $\lambda$ from 1 to 2 leads to reducing $V_{c r}$ up to 179 percent.

\section{CONCLUSION}

Calculative experiments showed, that the in influence of attenuation parameter $\beta$ of kernel to critical speed of the plate flutter in comparison with the viscosity $A$ parameter and singularity $\alpha$ were negligible that confirms the known conclusionexponential kernel of relaxation can't describe the material construction properties fully. The negligible reducing the singularity parameter leads to substantial reducing the critical speed of the flutter. Consequently taking in consideration of this effect at the aircraft construction projecting has great 
importance, as the less the singularity parameter of the material, the higher intensity of dissipative processes in these constructions.

\section{REFERENCES}

[1] Fung, Y.C., "Some recent contributions to panel flutter research." AIAA J. 1, 1963, pp.898-909.

[2] Fung, Y.C., "A summary of the theories and experiments on panel flutter, " Guggenheim Aeronautical Lab., California Institute of Technology, Air Force Office of Scientific Research TN 60-224 (may 1960).

[3] Dowell, E.H. and Voss, H.M. "Experimental and theoretical panel flutter studies in the Mach number range 1.0 to 5.0 , “ AIAA J. 3, 1965, pp. 2292-2304.

[4] Bolotin, V.V., Non-conservative Problems of the Theory of Elastic Stability, Moscow. 1961.p. 340. (in Russian).

[5] Volmir, A.S., Stability of Deformable Systems, Nauka, Moscow,1967. p. 768. (in Russian).

[6] Dowell, E.H., "Panel Flutter: A Review of the Aeroelastic stability of Plates and Shells," AIAA Journal, Vol.8, No2, 1970, pp.385-399.

[7] Bogdanovich, A.E. Nonlinear Problems of dynamic of cylindrical composite shells. Riga: Zinathe.-1987 (in Russian).

[8] Tamuzs, V.P. and Teters, G.A.," Problem of mechanics of Composite Materials, " Mechanics of Composite Materials J. N 1, 1979, pp. 34-45 (in Russian).

[9] Badalov, F. B., Eshmatov, H., “ One method of solution of system of Integra - differential the problems of viscoelasticity," Journal of Applied Mathematics and Mechanics. Moscow. Vol. 51, N 5. 1987, pp. 867-871. (in Russian).

[10] Khudayarov, B.A., Nonlinear flutter of the viscoelastic plates and cylindrical panels. Avtoreferat of diss. On candidate physicsmathematics science. Tashkent. Uzbekistan. 1998. p. 19. (in Russian).

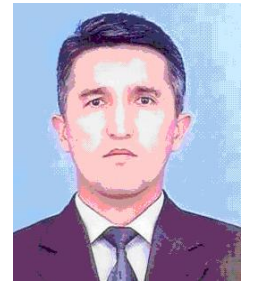

Khudayarov Bakhtiyar Alimovich

Associate of Professor of chair "Higher mathematics" of Tashkent institute of irrigation and melioration. Academic degree and status: Candidate of physicmathematics science, assistantprofessor. Year of birth: 1966.

Address: Chilanzar -16 -24-56, Tashkent, 700135, Uzbekistan. Phone: (998712) 76-04-02 (home), (99871) 137-09-86 (business).

E-mail: bakht-flpo@yandex.ru

Field of research: panel flutter and classic flutter. 